\title{
WHEN IS $C(X)$ POLYNOMIALLY IDEAL?
}

\author{
KARIM BOULABIAR AND SAMIR SMITI
}

\begin{abstract}
Let $\mathbb{A}$ be a commutative $f$-algebra with unit. The sets of all ideals in $\mathbb{A}$ and all intersections of maximal ideals in $\mathbb{A}$ are denoted by $\mathfrak{I}(\mathbb{A})$ and $\mathfrak{I M}(\mathbb{A})$, respectively. Whenever $\mathfrak{a} \in \mathfrak{I}(\mathbb{A})$, we say that $\mathbb{A}$ is polynomially $\mathfrak{a}$-ideal if, for every $f \in \mathbb{A}$ with $p(f) \in \mathfrak{a}$ for some non-zero polynomial $p(x)$, there is an $f_{0} \in \mathfrak{a}$ such that $p\left(f+f_{0}\right)=0$. We prove that if $\mathbb{A}$ is bounded inversion closed and $\mathfrak{a} \in \mathfrak{I M}(\mathbb{A})$, then $\mathbb{A}$ is polynomially $\mathfrak{a}$-ideal if and only if idempotents lift modulo a. This fact is based upon a systematic study of idempotent elements of an $f$-algebra. As a consequence, we show that, if $X$ is a Tychonoff space, then $C(X)$ is polynomially $\mathfrak{a}$-ideal for all $\mathfrak{a} \in \mathfrak{I}(C(X))$ if and only if $X$ is a $P$-space. Moreover, we prove that $C(X)$ is polynomially a-ideal for all $\mathfrak{a} \in \mathfrak{I M}(C(X))$ if and only if $X$ is strongly zero-dimensional. It turns out that this extends a theorem by Miers, namely, if $X$ is a compact Hausdorff space, then $C(X)$ is polynomially $\mathfrak{a}$-ideal for every uniformly closed ideal $\mathfrak{a}$ in $C(X)$ if and only if $X$ is totally disconnected.
\end{abstract}

1. Introduction. Let $\mathbb{A}$ be a commutative and associative real algebra with identity. The set of all ring ideals in $\mathbb{A}$ is denoted by $\mathfrak{I}(\mathbb{A})$ and its subset of all intersections of maximal ideals is denoted by $\mathfrak{I M}(\mathbb{A})$. Choose $\mathfrak{a} \in \mathfrak{I}(\mathbb{A})$. Modifying slightly a definition by Miers in [14], we say that $\mathbb{A}$ is polynomially $\mathfrak{a}$-ideal if, whenever $f \in \mathbb{A}$ and $p(f) \in \mathfrak{a}$ for some non-zero real polynomial $p(x)$, there is $g \in \mathfrak{a}$ for which $p(f+g)=0$. Now, let $C(X)$ be the commutative real algebra with identity of all real-valued continuous functions on a Tychonoff space $X$. It was proved by Miers himself (again in [14]) that a compact Hausdorff space $K$ is totally disconnected if and only if $C(K)$ is polynomially aideal for every uniformly closed ideal $\mathfrak{a}$ in $C(K)$. The observation to make here is that any uniformly closed ideal in $C(K)$ is in $\mathfrak{I} \mathfrak{M}(C(K))$.

2010 AMS Mathematics subject classification. Primary 06F25, 13A15, 46E25.

Keywords and phrases. Commutative $f$-algebra, idempotent element, idempotent lift modulo an ideal, polynomially ideal, bounded inversion closed, characteristic function, intersection of maximal ideals, completely regular space, lattice-ordered algebra.

Received by the editors on February 16, 2014, and in revised form on June 11, 2014.

DOI: $10.1216 / \mathrm{JCA}-2015-7-4-473$ 
Hence, a 'little more algebraic' version of the Miers theorem arises as follows. A necessary and sufficient condition for a compact Hausdorff space $K$ to be totally disconnected is that $C(K)$ is polynomially a-ideal for every $\mathfrak{a} \in \mathfrak{I M}(C(K))$.

This paper is in part developed around the following natural question. What happens if we leave the realm of compact Hausdorff spaces? Surprisingly enough, we shall prove that, if $X$ is a Tychonoff space, then $C(X)$ is polynomially a-ideal for every $\mathfrak{a} \in \mathfrak{I M}(C(X))$ if and only if $X$ is strongly zero-dimensional. The Miers theorem turns out to be a special case of our result. In spite of that, we shall get a 'new' characterization of $P$-spaces. Namely, $X$ is a $P$-space if and only if $C(X)$ is polynomially a-ideal for every $\mathfrak{a} \in \mathfrak{I}(C(X))$. Our approach relies heavily on a study of idempotent elements in an abstracted function algebra (also called $f$-algebra) in the sense of Birkhoff and Pierce [6]. More details seem to be in order.

Let $\mathbb{A}$ be a commutative $f$-algebra with identity, and let $\mathfrak{a}$ be a (ring) ideal in $\mathbb{A}$. We shall prove that, if $\mathbb{A}$ is semiprime and $f \in \mathbb{A}$, then $p(f)=0$ for some non-zero real polynomial $p(x)$ if and only if there exist pairwise different real numbers $\lambda_{1}, \ldots, \lambda_{n}$ and pairwise disjoint idempotent elements $e_{1}, \ldots, e_{n} \in \mathbb{A}$ such that $f=\lambda_{1} e_{1}+\cdots+\lambda_{n} e_{n}$. As a consequence, we will prove that, if $\mathbb{A}$ is bounded inversion closed and $\mathfrak{a} \in \mathfrak{I M}(\mathbb{A})$, then $\mathbb{A}$ is polynomially $\mathfrak{a}$-ideal if and only if idempotents lift modulo $\mathfrak{a}$, i.e., for every $f \in \mathbb{A}$ with $f^{2}-f \in \mathfrak{a}$, there exists $g \in \mathfrak{a}$ such that $f+g$ is an idempotent element of $\mathbb{A}$. In particular, if $\mathfrak{a} \in \mathfrak{I M}(C(X))$, then $C(X)$ is polynomially $\mathfrak{a}$-ideal if and only if, for each function $f \in C(X)$ which is idempotent modulo $\mathfrak{a}$ (i.e., $f^{2}-f \in \mathfrak{a}$ ) there is a function $g \in \mathfrak{a}$ such that $f+g$ is a characteristic function. As would be expected, this result is the key of our investigation in the $C(X)$ case.

We point out that our results and proofs are developed in the real case. Nevertheless, taking into consideration the Theory of Complex Vector Lattices and Complex $f$-Algebras as presented, e.g., in $[4,18]$, the whole content of the paper can be extended quite directly to the complex case.

We notice finally that a similar subject was investigated in [2] by Barnes within the (completely different) framework of Banach algebras. Also, Olsen [16] discusses the same problem for operator algebras. 
2. Results on idempotents in $f$-algebras. This section deals with idempotent elements of an $f$-algebra. By the way, we assume that the reader is familiar with the notion of vector lattices and lattice-ordered algebras. In this regard, we refer to the monographs $[5,12,17,19]$ for unexplained terminology and notation.

Beginning with the next paragraph, we shall impose the blanket assumption that any lattice-ordered algebra under consideration is real, commutative, and with identity 1 .

Following Birkhoff and Pierce [6], we call a lattice-ordered algebra $\mathbb{A}$ a function algebra (briefly, an $f$-algebra) if

$$
f \wedge g=0 \text { and } 0 \leq h \text { in } \mathbb{A} \text { imply }(f h) \wedge g=0 .
$$

It is well known that the lattice-ordered algebra $\mathbb{A}$ is an $f$-algebra if and only if 1 is weak order unit, that is, if $f \wedge 1=0$ in $\mathbb{A}$, then $f=0$ (see, e.g., [3, Corollary 1.10]). From now on, $\mathbb{A}$ stands for an $f$-algebra and $\mathbb{A}^{+}$denotes its positive cone.

It is readily checked that

$$
f g=0 \quad \text { whenever } \quad f, g \in \mathbb{A} \text { and } f \wedge g=0 .
$$

Hence, since

$$
f=f^{+}-f^{-} \text {for all } f \in \mathbb{A}
$$

where

$$
f^{+}=f \vee 0 \quad \text { and } \quad f^{-}=(-f) \vee 0
$$

we have

$$
f^{+} f^{-}=0 \quad \text { for all } f \in \mathbb{A} \text {. }
$$

It follows straightforwardly that $\mathbb{A}$ has positive squares. That is,

$$
f^{2}=\left(f^{+}\right)^{2}+\left(f^{-}\right)^{2} \in \mathbb{A}^{+} \text {for all } f \in \mathbb{A} \text {. }
$$

In particular, 1 is a positive element of $\mathbb{A}$. Furthermore, if $f, g \in \mathbb{A}^{+}$, then

$$
f^{2} \vee g^{2}=(f \vee g)^{2} \quad \text { and } \quad f^{2} \wedge g^{2}=(f \wedge g)^{2} .
$$

These equalities will be useful for later purposes, and their proofs can be found in [19, Theorem 142.1]. Now, put

$$
\operatorname{Idem}(\mathbb{A})=\left\{e \in \mathbb{A}: e^{2}=e\right\} .
$$


In other words, $\operatorname{Idem}(\mathbb{A})$ is the set of all idempotent elements of $\mathbb{A}$. Since $\mathbb{A}$ has positive squares, all idempotent elements of $\mathbb{A}$ are positive. These elementary properties will be used throughout the paper without further mention. Additional useful facts about idempotents elements of the $f$-algebra $\mathbb{A}$ are given next.

Lemma 2.1. Let $\mathbb{A}$ be an $f$-algebra and $u, v \in \operatorname{Idem}(\mathbb{A})$. Then the following hold.

(i) $1-u \in \operatorname{Idem}(\mathbb{A})$.

(ii) $u v=0$ if and only if $u \wedge v=0$.

(iii) $u-v \in \operatorname{Idem}(\mathbb{A})$ if and only if $u-v \in \mathbb{A}^{+}$.

Proof.

(i) We have

$$
(1-u)^{2}=1-2 u+u^{2}=1-2 u+u=1-u .
$$

So, $1-u \in \operatorname{Idem}(\mathbb{A})$, which is the desired result.

(ii) We already know that if $u \wedge v=0$, then $u v=0$. Conversely, suppose that $u v=0$. Hence,

$$
0 \leq u \wedge v=u^{2} \wedge v^{2}=(u \wedge v)^{2} \leq u v=0 .
$$

We get $u \wedge v=0$, as required.

(iii) If $u-v \in \operatorname{Idem}(\mathbb{A})$, then $u-v \in \mathbb{A}^{+}$. Conversely, from $u-v \in \mathbb{A}^{+}$, it follows that $u \leq v$ in $\mathbb{A}$ and so

$$
v=v^{2} \leq u v .
$$

Moreover, $1-u \in \operatorname{Idem}(\mathbb{A})$ (see (i)) so $1-u \in \mathbb{A}^{+}$. Hence, $0 \leq u \leq 1$, and thus $0 \leq u v \leq v$. This means that $u v=v$. We derive that

$$
(u-v)^{2}=u^{2}+v^{2}-2 u v=u+v-2 v=u-v .
$$

The proof is complete.

The following definitions play a key role in the sequel.

Definition 2.2. Let $\mathbb{A}$ be an $f$-algebra. 
(i) A subset $\left\{f_{1}, \ldots, f_{n}\right\}$ of $\mathbb{A}^{+}$is called a disjoint system if

$$
f_{i} \wedge f_{j}=0 \text { for all } i, j \in\{1, \ldots, n\} \text { with } i \neq j .
$$

(ii) A disjoint system $\left\{f_{1}, \ldots, f_{n}\right\}$ in $\mathbb{A}$ is said to be unital if $\sum_{i=1}^{n} f_{i}=$ 1.

We emphasize that all elements in a disjoint system in $\mathbb{A}$ are positive. Handy characterizations of unital disjoint systems in $\operatorname{Idem}(\mathbb{A})$ are provided next.

Lemma 2.3. Let $\mathbb{A}$ be an $f$-algebra and $e_{1}, \ldots, e_{n} \in \mathbb{A}$. Then the following are equivalent:

(i) $\left\{e_{1}, \ldots, e_{n}\right\}$ is a unital disjoint system in $\operatorname{Idem}(\mathbb{A})$.

(ii) $\sum_{i=1}^{n} e_{i}=1$ and $e_{i} e_{j}=0$ for all $i, j \in\{1, \ldots, n\}$ with $i \neq j$.

(iii) $\sum_{i=1}^{n} e_{i}=1$ and $e_{i} \in \operatorname{Idem}(\mathbb{A})$ for all $i \in\{1, \ldots, n\}$.

Proof.

(i) $\Rightarrow$ (ii). By definition, we have $\sum_{i=1}^{n} e_{i}=1$. Let $i, j \in\{1, \ldots, n\}$ with $i \neq j$. Then, $e_{i} \wedge e_{j}=0$ and so $e_{i} e_{j}=0$.

(ii) $\Rightarrow$ (iii). Let $i \in\{1, \ldots, n\}$ and observe that

$$
e_{i}=e_{i} \sum_{j=1}^{n} e_{j}=\sum_{j=1}^{n} e_{i} e_{j}=e_{i}^{2}+\sum_{j=1, j \neq i}^{n} e_{i} e_{j}=e_{i}^{2}
$$

We derive that $e_{i} \in \operatorname{Idem}(\mathbb{A})$, as desired.

(iii) $\Rightarrow$ (i). Choose $i, j \in\{1, \ldots, n\}$ with $i \neq j$. Then,

$$
e_{i}^{2}=e_{i}=e_{i} \sum_{k=1}^{n} e_{k}=\sum_{k=1}^{n} e_{i} e_{k}=e_{i}^{2}+\sum_{k=1, k \neq i}^{n} e_{i} e_{k}
$$

It follows that:

$$
0 \leq e_{i} e_{j} \leq \sum_{k=1, k \neq i}^{n} e_{i} e_{k}=0
$$

This, together with Lemma 2.1 (ii) completes the proof.

There is a way to extend a non-unital disjoint system of idempotent elements in $\mathbb{A}$ by adjoining a single vector in such a way that the 
resulting set is a unital disjoint system in $\mathbb{A}$. Details are given in the following lemma.

Lemma 2.4. Let $\mathbb{A}$ be an $f$-algebra and $\left\{e_{1}, \ldots, e_{n}\right\}$ a disjoint system in $\operatorname{Idem}(\mathbb{A})$. Then $\left\{e_{1}, \ldots, e_{n}, 1-\sum_{i=1}^{n} e_{i}\right\}$ is a unital disjoint system in $\mathbb{A}$.

Proof. Let $i \in\{1, \ldots, n\}$. Since $e_{i}^{2}=e_{i}$, we get:

$$
\left(1-\sum_{j=1}^{n} e_{j}\right) e_{i}=e_{i}-\sum_{j=1}^{n} e_{i} e_{j}=e_{i}-e_{i}^{2}=0 .
$$

We derive that the set

$$
\left\{e_{1}, \ldots, e_{n}, 1-\sum_{i=1}^{n} e_{i}\right\}
$$

fulfills condition Lemma 2.3 (ii). This gives the desired result.

As usual, the symbol $\mathbb{R}$ is used to indicate the field of all real numbers. Also, the principal ideal domain of all polynomials with coefficients in $\mathbb{R}$ is denoted by $\mathbb{R}[x]$. Our main results are also based upon the following lemma.

Lemma 2.5. Let $\mathbb{A}$ be an $f$-algebra and $\left\{e_{1}, \ldots, e_{n}\right\}$ a unital disjoint system in $\mathbb{A}$. Then

$$
p\left(\sum_{i=1}^{n} \lambda_{i} e_{i}\right)=\sum_{i=1}^{n} p\left(\lambda_{i}\right) e_{i} \quad \text { for all } \lambda_{1}, \ldots, \lambda_{n} \in \mathbb{R} \text { and } p \in \mathbb{R}[x] .
$$

Proof. Put

$$
f=\sum_{i=1}^{n} \lambda_{i} e_{i}
$$

and notice that

$$
f^{0}=1=\sum_{i=1}^{n} e_{i}=\sum_{i=1}^{n} \lambda_{i}^{0} e_{i}
$$


Moreover, let $m \in\{0,1,2, \ldots\}$, and assume that

$$
f^{m}=\sum_{i=1}^{n} \lambda_{i}^{m} e_{i}
$$

Using the equivalence (i) $\Leftrightarrow$ (ii) in Lemma 2.3, we obtain

$$
\begin{aligned}
f^{m+1} & =f^{m} f=\left(\sum_{i=1}^{n} \lambda_{i}^{m} e_{i}\right)\left(\sum_{i=1}^{n} \lambda_{i} e_{i}\right) \\
& =\sum_{i, j=1}^{n} \lambda_{i}^{m} \lambda_{j} e_{i} e_{j}=\sum_{i=1}^{n} \lambda_{i}^{m+1} e_{i}^{2} \\
& =\sum_{i=1}^{n} \lambda_{i}^{m+1} e_{i} .
\end{aligned}
$$

Thus, we have proved by induction that

$$
f^{m}=\sum_{i=1}^{n} \lambda_{i}^{m} e_{i} \quad \text { for all } m \in\{0,1,2, \ldots\} .
$$

Now, pick $p \in \mathbb{R}[x]$ with

$$
p(x)=\sum_{m=0}^{s} \alpha_{m} x^{m} .
$$

From (2.1), it follows that

$$
\begin{aligned}
p(f) & =\sum_{m=0}^{s} \alpha_{m} f^{m}=\sum_{m=0}^{s} \alpha_{m}\left(\sum_{i=1}^{n} \lambda_{i}^{m} e_{i}\right) \\
& =\sum_{m=0}^{s} \sum_{i=1}^{n} \alpha_{m} \lambda_{i}^{m} e_{i}=\sum_{i=1}^{n} \sum_{m=0}^{s} \alpha_{m} \lambda_{i}^{m} e_{i} \\
& =\sum_{i=1}^{n}\left(\sum_{m=0}^{s} \alpha_{m} \lambda_{i}^{m}\right) e_{i}=\sum_{i=1}^{n} p\left(\lambda_{i}\right) e_{i} .
\end{aligned}
$$

This finishes the proof.

Now, recall that the $f$-algebra $\mathbb{A}$ is said to be semiprime (or, reduced) if $\mathbb{A}$ contains no non-zero nilpotent elements. On the other hand, the 
zero-set of $p \in \mathbb{R}[x]$ is defined by

$$
Z(p)=\{\lambda \in \mathbb{R}: p(\lambda)=0\} .
$$

That is, $Z(p)$ is the set of all roots of $p$ in $\mathbb{R}$ (which can be empty, of course).

We have gathered at this point all the ingredients we need for the main result of this section.

Theorem 2.6. Let $\mathbb{A}$ be a semiprime $f$-algebra, $p \in \mathbb{R}[x]$ with $p \neq 0$, and $f \in \mathbb{A}$. Then the following are equivalent:

(i) $p(f)=0$.

(ii) There exist pairwise different $\lambda_{1}, \ldots, \lambda_{n} \in Z(p)$ such that

$$
\prod_{i=1}^{n}\left(f-\lambda_{i}\right)=0 .
$$

(iii) There exist pairwise different $\lambda_{1}, \ldots, \lambda_{n} \in Z(p)$ and a unital disjoint system $\left\{e_{1}, \ldots, e_{n}\right\}$ in $\mathbb{A}$ such that

$$
f=\sum_{i=1}^{n} \lambda_{i} e_{i} .
$$

Proof. (i) $\Rightarrow$ (ii). Put

$$
\mathcal{I}=\{s \in \mathbb{R}[x]: s(f)=0\} .
$$

Obviously, $\mathcal{I}$ is a principal ring ideal in $\mathbb{R}[x]$. From (i), it follows that $\mathcal{I} \neq\{0\}$, and so $\mathcal{I}$ has a unique monic generator $p_{f} \in \mathbb{R}[x]$. Actually, $p_{f}$ is the minimal polynomial of $f$, namely, if $s \in \mathbb{R}[x]$, then $s \in \mathcal{I}$ if and only if $p_{f}$ divides $s$. In particular, $p$ is a multiple of $p_{f}$ in $\mathbb{R}[x]$. We claim that there is no quadratic polynomial in the factorization of $p_{f}$ into irreducible elements of $\mathbb{R}[x]$. Otherwise, there would exist $\alpha, \beta \in \mathbb{R}$ with $\beta-\alpha^{2}>0$ and $q \in \mathbb{R}[x]$ with $q \neq 0$ such that

$$
p_{f}(x)=\left(x^{2}+2 \alpha x+\beta\right) q(x) .
$$

Observe that the inequalities

$$
0<\beta-\alpha^{2} \leq \beta-\alpha^{2}+(f+\alpha)^{2}=f^{2}+2 \alpha f+\beta
$$


hold in $\mathbb{A}$. It would follow that:

$$
\begin{aligned}
0 & <\left(\beta-\alpha^{2}\right)|q(f)| \\
& \leq\left(f^{2}+2 \alpha f+\beta\right)|q(f)| \\
& =\left|\left(f^{2}+2 \alpha f+\beta\right) q(f)\right|=0 .
\end{aligned}
$$

We conclude that $q(f)=0$, which contradicts the minimality of $p_{f}(x)$. Thus, $p_{f}(x)$ has no quadratic polynomial in its factorization into irreducible elements in $\mathbb{R}[x]$. Notice now that $\mathbb{R}[x]$ has no irreducible elements other than quadratic polynomials with negative discriminants and linear polynomials. It follows, in particular, that $Z\left(p_{f}\right)$ is non empty. Put

$$
Z\left(p_{f}\right)=\left\{\lambda_{1}, \ldots, \lambda_{n}\right\}
$$

(in particular, $\lambda_{i} \neq \lambda_{j}$ whenever $i \neq j$ in $\{1, \ldots, n\}$ ). As noticed above, $p_{f}$ divides $p$, and so

$$
\lambda_{1}, \ldots, \lambda_{n} \in Z(p)
$$

Moreover, there exist $\omega_{1}, \ldots, \omega_{n} \in\{1,2, \ldots\}$ such that

$$
p_{f}(x)=\prod_{i=1}^{n}\left(x-\lambda_{i}\right)^{\omega_{i}}
$$

Put $\omega=\max \left\{\omega_{i}: i \in\{1, \ldots, n\}\right\}$, and observe that

$$
\left(\prod_{i=1}^{n}\left(x-\lambda_{i}\right)\right)^{\omega}=p_{f}(x) \prod_{i=1}^{n}\left(x-\lambda_{i}\right)^{\omega-\omega_{i}} .
$$

Hence,

$$
\left(\prod_{i=1}^{n}\left(f-\lambda_{i}\right)\right)^{\omega}=p_{f}(f) \prod_{i=1}^{n}\left(f-\lambda_{i}\right)^{\omega-\omega_{i}}=0
$$

Since $\mathbb{A}$ is semiprime, we obtain

$$
\prod_{i=1}^{n}\left(f-\lambda_{i}\right)=0
$$

and (ii) follows. 
(ii) $\Rightarrow$ (iii). Define

$$
p(x)=\prod_{i=1}^{n}\left(x-\lambda_{i}\right)
$$

and

$$
p_{i}(x)=\frac{p(x)}{x-\lambda_{i}} \in \mathbb{R}[x] \text { for all } i \in\{1, \ldots, n\} .
$$

Clearly, 1 is the greatest common divisor of $p_{1}, \ldots, p_{n}$. Hence, there exist $q_{1}, \ldots, q_{n} \in \mathbb{R}[x]$ such that

$$
\sum_{i=1}^{n} p_{i} q_{i}=1
$$

(where we use the classical Bezout's theorem in $\mathbb{R}[x]$ ). Therefore, the equality

$$
\sum_{i=1}^{n} p_{i}(f) q_{i}(f)=1
$$

holds in $\mathbb{A}$. Put

$$
e_{i}=p_{i}(f) q_{i}(f) \quad \text { for all } i \in\{1, \ldots, n\} .
$$

Thus,

$$
\sum_{i=1}^{n} e_{i}=1
$$

Furthermore, choose $i, j \in\{1, \ldots, n\}$ with $i \neq j$. Clearly, $p_{f}$ divides $p_{i} p_{j}$, and so

$$
p_{i}(f) p_{j}(f)=0
$$

Hence,

$$
e_{i} e_{j}=p_{i}(f) q_{i}(f) p_{j}(f) q_{j}(f)=0 .
$$

Combining Lemma 2.3, (2.2) and (2.3), we get

$$
e_{i}^{2}=e_{i} \quad \text { for all } i \in\{1, \ldots, n\} .
$$

Consequently, if $i \in\{1, \ldots, n\}$, then

$$
\left(f-\lambda_{i}\right) e_{i}=\left(f-\lambda_{i}\right) p_{i}(f) q_{i}(f)=p(f) q_{i}(f)=0,
$$


and so $f e_{i}=\lambda_{i} e_{i}$. Whence,

$$
f=f \sum_{i=1}^{n} e_{i}=\sum_{i=1}^{n} f e_{i}=\sum_{i=1}^{n} \lambda_{i} e_{i}
$$

which leads to the desired assertion.

(iii) $\Rightarrow$ (i). We have

$$
p\left(\lambda_{i}\right)=0 \text { for all } i \in\{1, \ldots, n\} .
$$

According to Lemma 2.5, we obtain

$$
p(f)=\sum_{i=1}^{n} p\left(\lambda_{i}\right) e_{i}=0,
$$

which finishes the proof of the theorem.

A version of Theorem 2.6 was obtained in [7] for the special case of orthomorphisms on archimedean vector lattices. Moreover, the above proof has to be slightly modified in the complex case. Indeed, we do not have to prove that $p_{f}$ has no irreducible quadratic divisors. By the way, this is the only place where we have to be a little careful moving from the real case to the complex case.

3. Polynomially a-ideal $f$-algebras. The main objective of the present section is to give a convenient necessary and sufficient condition for an $f$-algebra $\mathbb{A}$ to be polynomially $\mathfrak{a}$-ideal, where $\mathfrak{a}$ is a semiprime $\ell$-ideal in $\mathbb{A}$. Let us discuss some of the relevant notions.

First, we label the definition around which this paper is developed.

Definition 3.1. Let $\mathfrak{a}$ be a ring ideal in an $f$-algebra $\mathbb{A}$. Then $\mathbb{A}$ is said to be polynomially a-ideal if for every $f \in \mathbb{A}$ for which $p(f) \in \mathfrak{a}$ for some $p \in \mathbb{R}[x]$ with $p \neq 0$ there exists $f_{0} \in \mathfrak{a}$ such that $p\left(f_{0}+f\right)=0$.

Now, recall that $\mathbb{A}$ is an $f$-algebra. A ring ideal $\mathfrak{a}$ in $\mathbb{A}$ is said to be convex if $\mathfrak{a}$ contains with any $f$ all $g$ such that $|g| \leq|f|$. We call an $\ell$-ideal in $\mathbb{A}$ any convex ring ideal in $\mathbb{A}$. Clearly, any $\ell$-ideal $\mathfrak{a}$ in $\mathbb{A}$ is a sublattice of the underlying lattice of $\mathbb{A}$. In particular, the $\ell$-ideal $\mathfrak{a}$ is closed under finite suprema. The next lines deal with quotient $f$-algebras. 
Let $\mathfrak{a}$ be an $\ell$-ideal in $\mathbb{A}$. The residue class of any $f \in \mathbb{A}$ in the quotient real algebra $A / \mathfrak{a}$ is denoted by $\mathfrak{a}(f)$. Of course, $\mathbb{A} / \mathfrak{a}$ is again commutative with $\mathfrak{a}(1)$ as the identity. The canonical surjection, denoted again by $\mathfrak{a}$, from $\mathbb{A}$ onto $\mathbb{A} / \mathfrak{a}$ is an algebra homomorphism. That is,

$$
\mathfrak{a}(\lambda f+g h)=\lambda \mathfrak{a}(f)+\mathfrak{a}(g) \mathfrak{a}(h) \quad \text { for all } \lambda \in \mathbb{R} \text { and } f, g, h \in \mathbb{A} .
$$

As $\mathfrak{a}$ is an $\ell$-ideal, the algebra $\mathbb{A} / \mathfrak{a}$ can be endowed with an ordering by putting

$$
\mathfrak{a}(f) \leq \mathfrak{a}(g) \quad \text { if and only if } h \leq g-f \text { for some } h \in \mathfrak{a} .
$$

Actually, $\mathbb{A} / \mathfrak{a}$ is an $f$-algebra with respect to this ordering. Furthermore, $\mathfrak{a}$ is a lattice homomorphism from $\mathbb{A}$ onto $\mathbb{A} / \mathfrak{a}$, which means that

$$
\mathfrak{a}(f \vee g)=\mathfrak{a}(f) \vee \mathfrak{a}(g) \quad \text { for all } f, g \in \mathbb{A}
$$

(more information can be found in $[\mathbf{5 , 1 2}]$ ). An $\ell$-ideal $\mathfrak{a}$ in $\mathbb{A}$ is said to be semiprime if $f \in \mathbb{A}$ and $f^{n} \in \mathfrak{a}$ for some $n \in\{1,2, \ldots\}$ imply $f \in \mathfrak{a}$. Obviously, the $\ell$-ideal $\mathfrak{a}$ is semiprime if and only if the $f$-algebra $\mathbb{A} / \mathfrak{a}$ is semiprime.

Now, let $\mathfrak{a}$ be an ideal in the commutative ring $\mathbb{A}$. We say that idempotents lift modulo $\mathfrak{a}$ if, given $f \in \mathbb{A}$ with $f^{2}-f \in \mathfrak{a}$, there exists $f_{0} \in \mathfrak{a}$ such that $f_{0}+f \in \operatorname{Idem}(\mathbb{A})$. We are thus in position to prove one of the main results of this paper, which gives a necessary and sufficient condition for the $f$-algebra $\mathbb{A}$ to be polynomially $\mathfrak{a}$-ideal, where $\mathfrak{a}$ is a semiprime $\ell$-ideal in $\mathbb{A}$.

Theorem 3.2. Let $\mathfrak{a}$ be a semiprime $\ell$-ideal in an $f$-algebra $\mathbb{A}$. Then $\mathbb{A}$ is polynomially $\mathfrak{a}$-ideal if and only if idempotents lift modulo $\mathfrak{a}$.

Proof. Suppose that $\mathbb{A}$ is polynomially a-ideal. Let $f \in \mathbb{A}$, and assume that $f^{2}-f \in \mathfrak{a}$. Put

$$
p(x)=x^{2}-x \in \mathbb{R}[x],
$$

and notice that $p(f) \in \mathfrak{a}$. Therefore, there exists $f_{0} \in \mathfrak{a}$ such that $p\left(f_{0}+f\right)=0$. That is, $f_{0}+f \in \operatorname{Idem}(\mathbb{A})$, as required.

Conversely, assume that, for every $f \in \mathbb{A}$ with $f^{2}-f \in \mathfrak{a}$, there exists $f_{0} \in \mathfrak{a}$ such that $f_{0}+f \in \operatorname{Idem}(\mathbb{A})$. Since $\mathfrak{a}$ is a semiprime 
$\ell$-ideal, the $f$-algebra $\mathbb{A} / \mathfrak{a}$ is semiprime. Let $f \in \mathbb{A}$, and assume that $p(f) \in \mathfrak{a}$ for some non-zero polynomial $p \in \mathbb{R}[x]$. It follows quickly that

$$
p(\mathfrak{a}(f))=0 \quad \text { in } \mathbb{A} / \mathfrak{a} .
$$

By Theorem 2.6, there exist $\lambda_{1}, \ldots, \lambda_{n} \in Z(p)$ and $e_{1}, \ldots, e_{n} \in \mathbb{A}$ such that

(3.1) $\quad\left\{\mathfrak{a}\left(e_{1}\right), \ldots, \mathfrak{a}\left(e_{n}\right)\right\} \quad$ is a unital disjoint system in $\mathbb{A} / \mathfrak{a}$

and

$$
\mathfrak{a}(f)=\sum_{i=1}^{n} \lambda_{i} \mathfrak{a}\left(e_{i}\right)
$$

Lemma 2.3 and (3.1) show that

$$
\mathfrak{a}\left(e_{i}\right) \in \operatorname{Idem}(\mathbb{A}) \quad \text { for all } i \in\{1, \ldots, n\} .
$$

Thanks to the hypothesis, we may assume, without loss of generality, that

$$
e_{i} \in \operatorname{Idem}(\mathbb{A}) \quad \text { for all } i \in\{1, \ldots, n\}
$$

Combining (3.1) and Lemma 2.3, we immediately get

$$
e_{i} e_{j} \in \mathfrak{a} \quad \text { for all } i, j \in\{1, \ldots, n\} \text { with } i \neq j \text {. }
$$

Now, define

$$
u_{i}=e_{i} \wedge \bigvee_{\substack{j=1 \\ j \neq i}}^{n} e_{j} \quad \text { for all } i \in\{1, \ldots, n\}
$$

Let $i \in\{1, \ldots, n\}$. Then observe that

$$
0 \leq u_{i}^{2} \leq e_{i} \bigvee_{\substack{j=1 \\ j \neq i}}^{n} e_{j}=\bigvee_{\substack{j=1 \\ j \neq i}}^{n} u_{i} e_{j} \in \mathfrak{a}
$$

(where we use (3.4)). It follows that $u_{i}^{2} \in \mathfrak{a}$ because $\mathfrak{a}$ is an $\ell$-ideal in 
$\mathbb{A}$ and then $u_{i} \in \mathfrak{a}$ since $\mathfrak{a}$ is semi-prime. Furthermore,

$$
\begin{aligned}
u_{i}^{2} & =\left(e_{i} \wedge \bigvee_{\substack{j=1 \\
j \neq i}}^{n} e_{j}\right)^{2}=e_{i}^{2} \wedge \bigvee_{\substack{j=1 \\
j \neq i}}^{n} e_{j}^{2} \\
& =e_{i} \wedge \bigvee_{\substack{j=1 \\
j \neq i}}^{n} e_{j}=u_{i} .
\end{aligned}
$$

This yields that $u_{i} \in \operatorname{Idem}(\mathbb{A})$. Also, from

$$
0 \leq u_{i}=e_{i} \wedge \bigvee_{\substack{j=1 \\ j \neq i}}^{n} e_{j} \leq e_{i}
$$

and Lemma 2.1 (iii), it follows that

$$
v_{i}=e_{i}-u_{i} \in \operatorname{Idem}(\mathbb{A}) .
$$

Notice here that, if $i \in\{1, \ldots, n\}$, then

$$
\mathfrak{a}\left(v_{i}\right)=\mathfrak{a}\left(e_{i}\right)-\mathfrak{a}\left(u_{i}\right)=\mathfrak{a}\left(e_{i}\right)
$$

(since $u_{i} \in \mathfrak{a}$ ). On the other hand, if $i \neq j$ in $\{1, \ldots, n\}$, then

$$
\begin{aligned}
0 & \leq v_{i} \wedge v_{j}=\left(e_{i}-u_{i}\right) \wedge\left(e_{j}-u_{j}\right) \\
& =\left(e_{i}-\left(e_{i} \wedge \bigvee_{\substack{k=1 \\
k \neq i}}^{n} e_{k}\right)\right) \wedge\left(e_{j}-\left(e_{j} \wedge \bigvee_{\substack{k=1 \\
k \neq j}}^{n} e_{k}\right)\right) \\
& =\left(e_{i}-\bigvee_{\substack{k=1 \\
k \neq i}}^{n} e_{k}\right)^{+} \wedge\left(e_{j}-\bigvee_{\substack{k=1 \\
k \neq j}}^{n} e_{k}\right)^{+} \\
& =\left(\bigwedge_{\substack{n=1 \\
k \neq i}}^{n}\left(e_{i}-e_{k}\right)\right)^{+} \wedge\left(\bigwedge_{\substack{n=1 \\
k \neq j}}^{n}\left(e_{j}-e_{k}\right)\right)^{+} \\
& \leq\left(e_{i}-e_{j}\right)^{+} \wedge\left(e_{j}-e_{i}\right)^{+}=0 .
\end{aligned}
$$

Accordingly,

$$
v_{i} \wedge v_{j}=0 .
$$


Denote

$$
v_{n+1}=1-\sum_{i=1}^{n} v_{i}
$$

Combining (3.5), (3.7) and Lemma 2.4, we derive that $\left\{v_{1}, \ldots, v_{n+1}\right\}$ is a unital disjoint system in $\mathbb{A}$. Putting $\lambda_{n+1}=\lambda_{n}$, we obtain, by Lemma 2.5, that

$$
p\left(\sum_{i=1}^{n+1} \lambda_{i} v_{i}\right)=\sum_{i=1}^{n+1} p\left(\lambda_{i}\right) v_{i}=0 .
$$

Moreover, (3.1) and (3.6) imply that $v_{n+1} \in \mathfrak{a}$. Indeed,

$$
\begin{aligned}
\mathfrak{a}\left(v_{n+1}\right) & =\mathfrak{a}\left(1-\sum_{i=1}^{n} v_{i}\right)=\mathfrak{a}(1)-\sum_{i=1}^{n} \mathfrak{a}\left(v_{i}\right) \\
& =\mathfrak{a}(1)-\sum_{i=1}^{n} \mathfrak{a}\left(e_{i}\right)=0 \quad \text { in } \mathbb{A} / \mathfrak{a} .
\end{aligned}
$$

Furthermore, from (3.2), it follows that there exists $g \in \mathfrak{a}$ such that

$$
f=g+\sum_{i=1}^{n} \lambda_{i} e_{i}=g+\sum_{i=1}^{n} \lambda_{i} v_{i}+\sum_{i=1}^{n} \lambda_{i} u_{i}
$$

Put

$$
f_{0}=-g-\sum_{i=1}^{n} \lambda_{i} u_{i}+\lambda_{n+1} v_{n+1}
$$

(recall that $\lambda_{n+1}=\lambda_{n}$ ). Clearly, $f_{0} \in \mathfrak{a}$ because $g, v_{n+1}, u_{i} \in \mathfrak{a}$ for all $i \in\{1, \ldots, n\}$. Finally,

$$
f_{0}+f=-g-\sum_{i=1}^{n} \lambda_{i} u_{i}+\lambda_{n+1} v_{n+1}+g+\sum_{i=1}^{n} \lambda_{i} e_{i}=\sum_{i=1}^{n+1} \lambda_{i} v_{i}
$$

and so

$$
p\left(f_{0}+f\right)=p\left(\sum_{i=1}^{n+1} \lambda_{i} v_{i}\right)=0
$$

(where we use (3.8)). This finishes the proof of the theorem.

In what follows, we shall discuss a particular case of Theorem 3.2. First, recall that the $f$-algebra $\mathbb{A}$ is said to be bounded inversion closed 
if

$1 \leq f$ in $\mathbb{A}$ implies that $f$ is a unit (i.e., has an inverse) in $\mathbb{A}$.

It was proved by Henriksen, Isbell and Johnson in [10, Lemma 1.1] that a $\Phi$-algebra (i.e., an archimedean $f$-algebra with identity) is bounded inversion closed if and only if any maximal ring ideal is convex (and thus an $\ell$-ideal). Actually, nowhere in the proof is it used that the $f$-algebra under consideration is archimedean or has an identity. It follows that it goes over any $f$-algebra.

Proposition 3.3. An $f$-algebra $\mathbb{A}$ is bounded inversion closed if and only if every maximal ring ideal in $\mathbb{A}$ is an $\ell$-ideal.

The following consequence of Theorem 3.2 and Proposition 3.3 is the last result of this section. It will be extremely useful for the concrete situation discussed in the final section of this work.

Corollary 3.4. Let $\mathbb{A}$ be a bounded inversion closed $f$-algebra and $\mathfrak{a} \in \mathfrak{I M}(\mathbb{A})$. Then $\mathbb{A}$ is polynomially $\mathfrak{a}$-ideal if and only if idempotents lift modulo a.

Proof. Taking into account Theorem 3.2, it suffices to observe that $\mathfrak{a}$ is a semiprime $\ell$-ideal in $\mathbb{A}$. Since any maximal ring ideal in $\mathbb{A}$ is prime, $\mathfrak{a}$ turns out to be an intersection of prime ring ideals and then semiprime. Moreover, from Proposition 3.3, it follows that any maximal ring ideal in $\mathbb{A}$ is an $\ell$-ideal. But then $\mathfrak{a}$ is again an $\ell$-ideal (as an intersection of $\ell$-ideals) and the corollary follows.

4. Polynomially ideal $C(X)$-type $f$-algebras. In this section, we shall use by and large the great text [9] by Gillman and Jerison as the main reference on $C(X)$-type $f$-algebras, unless it conflicts with the by now standard notation and terminology. For instance, strongly zerodimensional spaces are called in [9] just zero-dimensional spaces. We use the term zero-dimensional for a space with a base of clopen sets.

Let $C(X)$ indicate the commutative $f$-algebra of all continuous real-valued functions on a Tychonoff space (i.e., a completely regular 
Hausdorff space) $X$. The constant function $\mathbf{1} \in C(X)$ defined by

$$
\mathbf{1}(x)=1 \quad \text { for all } x \in X
$$

is the identity of $C(X)$. Moreover, the characteristic function of a subset $Y$ of $X$ is denoted by $\mathbf{1}_{Y}$. Hence, $e \in C(X)$ is idempotent if and only if $e=\mathbf{1}_{V}$ for some clopen set $V$ in $X$. Recall that $\mathfrak{I M}(C(X))$ denotes the set of all intersections of maximal ring ideals in $C(X)$. Hence, a ring ideal $\mathfrak{a}$ in $C(X)$ is in $\mathfrak{I M}(C(X))$ if and only if there exists a family $\left\{\mathfrak{m}_{i}\right\}_{i \in I}$ of maximal ring ideals in $C(X)$ such that $\mathfrak{a}=\underset{i \in I}{\cap} \mathfrak{m}_{i}$.

Let us give the $C(X)$-version of Corollary 3.4.

Lemma 4.1. Let $X$ be a Tychonoff space and $\mathfrak{a} \in \mathfrak{I M}(C(X))$. Then $C(X)$ is polynomially $\mathfrak{a}$-ideal if and only if idempotents lift modulo $\mathfrak{a}$.

Proof. This follows directly from Corollary 3.4 because $C(X)$ is a bounded inversion closed $f$-algebra.

In other words, if $\mathfrak{a} \in \mathfrak{I M}(C(X))$, then $C(X)$ is polynomially $\mathfrak{a}$-ideal if and only if, for every $f \in C(X)$ with $f^{2}-f \in \mathfrak{a}$, there exists $f_{0} \in \mathfrak{a}$ such that $f_{0}+f$ is a characteristic function in $C(X)$.

Recall that an element in a ring is said to be clean if it may be written as a sum of a unit and idempotent. The ring itself is called clean if every element is clean. Moreover, let $\mathfrak{I M}_{0}(C(X))$ denote the set of all intersections of fixed maximal ideals in $C(X)$. Of course, $\mathfrak{I M}_{0}(C(X))$ is a subset of $\mathfrak{I M}(C(X))$.

We arrive at this point to the main result of this paper (the second and fourth assertions were added after a recommendation by the referee).

Theorem 4.2. Let $X$ be a Tychonoff space. Then the following are equivalent:

(i) $C(X)$ is polynomially $\mathfrak{a}$-ideal for all $\mathfrak{a} \in \mathfrak{I M}(C(X))$.

(ii) $C(X)$ is polynomially $\mathfrak{a}$-ideal for all $\mathfrak{a} \in \mathfrak{I M}_{0}(C(X))$.

(iii) $X$ is strongly zero-dimensional.

(iv) $C(X)$ is a clean ring. 
Proof.

(i) $\Rightarrow$ (ii). Trivial.

(ii) $\Rightarrow$ (iii). Let $f, g \in C(X)$ such that $Z(f)$ and $Z(g)$ are two disjoint zero-sets in $X$. We claim that $Z(f)$ and $Z(g)$ are separated by a partition of $X$ (for partitions, see [9, Section 16.12]). To this end, observe that the function $|f|+|g|$ does not vanish and so a function $e \in C(X)$ can be defined by

$$
e=\frac{|f|}{|f|+|g|} \text {. }
$$

Obviously,

$$
e[Z(f)]=\{0\} \quad \text { and } \quad e[Z(g)]=\{1\} .
$$

Define

$$
\mathfrak{a}=\{f \in C(X): f(x)=0 \quad \text { for all } x \in Z(f) \cup Z(g)\} .
$$

It is not hard to see that $\mathfrak{a} \in \mathfrak{I M}_{0}(C(X))$. Furthermore, a simple calculation reveals that $e^{2}-e \in \mathfrak{a}$. Since $C(X)$ is polynomially a-ideal and $a \in \mathfrak{I M}(C(X))$, Lemma 4.1 shows that there exists $e_{0} \in \mathfrak{a}$ such that $e_{0}+e$ is a characteristic function in $C(X)$. Put $V=\left(e_{0}+e\right)^{\leftarrow}(1)$, and observe that $V$ is a clopen set in $X$ (actually, $e_{0}+e=\mathbf{1}_{V}$ ). If $x \in Z(f)$, then $e_{0}(x)=e(x)=0$, and so

$$
\left(e_{0}+e\right)(x)=e_{0}(x)+e(x)=0 .
$$

Hence, $Z(f) \subset W=X-V$. Analogously, if $x \in Z(g)$, then $e_{0}(x)=0$ and $e(x)=1$. Thus,

$$
\left(e_{0}+e\right)(x)=e_{0}(x)+e(x)=1 .
$$

This yields that $Z(g) \subset V$. Consequently, $Z(f)$ and $Z(g)$ are separated by a partition of $X$, as required. But then [9, Theorem 16.17] implies that $X$ is strongly zero-dimensional.

(iii) $\Rightarrow$ (iv). See [1, Theorem 2.5] or [13, Theorem 13].

(iv) $\Rightarrow$ (i). Combining Corollary 1.3 and Proposition 1.8 in [15], we derive that idempotents lift modulo any ideal $\mathfrak{a}$ in $C(X)$. Thus, idempotents lift modulo any $\mathfrak{a}$ in $\mathfrak{I M}(C(X))$. This, together with Lemma 4.1 leads directly to (i) and completes the proof. 
Recall that a compact Hausdorff space is strongly zero-dimensional if and only if it is zero-dimensional. We get the following result, which was suggested to us by the referee.

Corollary 4.3. Let $X$ be a Tychonoff space. Then $C(X)$ is polynomially $\mathfrak{a}$-ideal for all $\mathfrak{a} \in \mathfrak{I M}(C(X))$ if and only if $C^{*}(X)$ is polynomially $\mathfrak{a}$-ideal for all $\mathfrak{a} \in \mathfrak{I M}\left(C^{*}(X)\right)$.

Proof. Applying Theorem 4.2 to the Stone-Čech compactification $\beta X$ of $X$, we derive that $C^{*}(X)=C(\beta X)$ is polynomially a-ideal for all $\mathfrak{a} \in \mathfrak{I M}\left(C^{*}(X)\right)$ if and only if $\beta X$ is (strongly) zero-dimensional. Thus, $C^{*}(X)$ is polynomially $\mathfrak{a}$-ideal for all $\mathfrak{a} \in \mathfrak{I M}\left(C^{*}(X)\right)$ if and only if $X$ is strongly zero-dimensional. Theorem 4.2 completes the proof.

The result by Miers mentioned in the introduction becomes a special case of Theorem 4.2 .

Corollary 4.4. (Miers $[\mathbf{1 4}$, Theorem 1]). Let $X$ be a compact Hausdorff space. Then $C(X)$ is polynomially a-ideal for every uniformly closed ideal if and only if $X$ is totally disconnected.

Proof. Since $X$ is compact, from [9, Problem 7Q] it follows that $\mathfrak{I M}(C(X))$ is the set uniformly closed ideals in $C(X)$. Moreover, by [9, Theorem 16.17], the space $X$ is totally disconnected if and only if $X$ is strongly zero-dimensional. The rest follows directly from the previous theorem.

Recall that $\mathfrak{I}(C(X))$ is the set of all ring ideals in $C(X)$. As noticed earlier, it is natural to ask when $C(X)$ is polynomially a-ideal for all $\mathfrak{a} \in \mathfrak{I}(C(X))$. The last result of this paper answers this question.

Corollary 4.5. Let $X$ be a Tychonoff space. Then $C(X)$ is polynomially $\mathfrak{a}$-ideal for all $\mathfrak{a} \in \mathfrak{I}(C(X))$ if and only if $X$ is a $P$-space.

Proof. Suppose that $C(X)$ is polynomially a-ideal for every $\mathfrak{a} \in$ $\mathfrak{I}(C(X))$. Let $f \in C(X)$ and $\mathfrak{a}$ denote the principal ideal in $C(X)$ generated by $f^{2}$. In particular, $p(f) \in \mathfrak{a}$, where $p(x)=x^{2}$. Since $C(X)$ is polynomially a-ideal, there is $f_{0} \in \mathfrak{a}$ with

$$
\left(f+f_{0}\right)^{2}=p\left(f+f_{0}\right)=\mathbf{0} .
$$


Hence, $f=-f_{0} \in \mathfrak{a}$ and so $f=f^{2} g$ for some $g \in C(X)$. This, together with [9, Problem $4 \mathrm{~J}]$ yields that $X$ is a $P$-space, indeed.

Conversely, suppose that $X$ is a $P$-space. Hence, $X$ is strongly zerodimensional (see [9, Problems 4K, 16O]). By Theorem 4.2, we infer that $C(X)$ is polynomially $\mathfrak{a}$-ideal for all $\mathfrak{a} \in \mathfrak{I M}(C(X))$. Moreover, the equality $\mathfrak{I}(C(X))=\mathfrak{I M}(C(X))$ holds because $X$ is a $P$-space (see again [9, Problem $4 \mathrm{~J}])$. It follows that $C(X)$ is polynomially a-ideal for all $\mathfrak{a} \in \mathfrak{I}(C(X))$, and the proof is complete.

\section{REFERENCES}

1. F. Azarpanah, When is $C(X)$ a clean ring?, Acta Math. Hung. 94 (2002), $53-58$.

2. B.A. Barnes, Algebraic elements of a Banach algebra modulo an ideal, Pac. J. Math. 117 (1985), 219-231.

3. S.J. Bernau and C.B. Huijsmans, Almost $f$-algebras and d-algebras, Math. Proc. Cambr. Philos. Soc. 107 (1990), 287-308.

4. F. Beukers, C.B. Huijsmans and B. de Pagter, Unital embedding and complexifications of $f$-algebras, Math. Z. 18 (1983), 131-144.

5. A. Bigard, K. Keimel and S. Wolfenstein, Groupes et Anneaux Réticulés, Lect. Notes Math. 608, Springer-Verlag, Berlin, 1977.

6. G. Birkhoff and R.S. Pierce, Lattice-ordered rings, An. Acad. Brasil. Ci. 28 (1956), 41-69.

7. K. Boulabiar, G. Buskes and G. Sirotkin, Algebraic order bounded disjointness preserving operators and strongly diagonal operators, Int. Equat. Oper. Theor. $\mathbf{5 4}$ (2006), 9-31.

8. L. Gillman, M. Henriksen and M. Jerison, On a theorem of Gelfand and Kolmogoroff concerning maximal ideals in rings of continuous functions, Proc. Amer. Math. Soc. 5 (1954), 447-455. 1976 .

9. L. Gillman and M. Jerison, Rings of continuous functions, Springer, Berlin,

10. M. Henriksen, J.R. Isbell and D.G. Johnson, Residue class fields of latticeordered algebras, Fund. Math. 50 (1961), 107-117.

11. E. Hewitt, Rings of real-valued continuous functions I, Trans. Amer. Math. Soc. 64 (1948), 45-99.

12. W.A.J. Luxemburg and A.C. Zaanen, Riesz spaces I, North-Holland, Amsterdam, 1971.

13. W.W. McGovern, Clean semiprime $f$-rings with bounded inversion, Comm. Alg. 31 (2003), 3295-3304.

14. C.R. Miers, Polynomially ideal $C^{*}$-algebras, Amer. Math. J. 98 (1976), 165170 . 
15. W.K. Nicholson, Lifting idempotents and exchange rings, Trans. Amer. Math. Soc. 229 (1977), 269-278.

16. C.L. Olsen, A structure theorem for polynomially compact operator, Amer. J. Math. 93 (1971), 686-698.

17. S.A. Steinberg, Lattice-ordered rings and modules, Springer, New York, 2010.

18. A.C. Zaanen, Introduction to operator theory in Riesz spaces, Springer, Berlin, 1997.

19. , Riesz spaces II, North-Holland, Amsterdam, 1983.

Research Laboratory of Algebra, Topology, Arithmetic, and Order, Department of Mathematics, Faculty of Mathematical, Physical and Natural Sciences of Tunis, Tunis-El Manar University, 2092-El Manar, Tunisia

Email address: karim.boulabiar@ipest.rnu.tn

Research Laboratory of Algebra, Topology, Arithmetic, and Order, Department of Mathematics, Faculty of Mathematical, Physical and Natural Sciences of Tunis, Tunis-El Manar University, 2092-El Manar, Tunisia

Email address: Smiti.samir@yahoo.fr 